\title{
Comparison of the hadronic structures in structure function measurements of the proton, photon and diffractive exchange
}

\author{
Daniel van Asseldonk, Martin Erdmann, Tatsiana Klimkovich*, Michael Nienhaus \\ RWTH Aachen University, Physics Institute $3 A$ \\ E-mail: \\ daniel.van.asseldonkerwth-aachen.de, \\ erdmannephysik.rwth-aachen.de \\ klimkovichephysik.rwth-aachen.de \\ Michael.Nienhauserwth-aachen.de
}

In the last several years a large number of new measurements has been performed in the area of hadronic structures. Most of these measurements come from HERA experiments. Very often, many data points and multiple parameter descriptions make reading of physics messages from the data not obvious. An intuitive description of structure functions can be achieved by employing fits based on the logarithmic $Q^{2}$-dependence of the structure function measurements. This allows to compare simultaneously the $x$-dependencies of the hadronic structures and the interaction dynamics of the proton, the photon, and colour singlet exchange. This contribution is the extension of the publication Phys. Lett. B488 (2000) 131 using new data.

XVIII International Workshop on Deep-Inelastic Scattering and Related Subjects, DIS 2010 April 19-23, 2010

Firenze, Italy

\footnotetext{
* Speaker.
} 


\section{Analysis Method}

The logarithmic dependence of hadronic structures on the resolution scale $Q^{2}$ predicted by the QCD evolution equations has led to an idea of fitting all available data of the structure function $F_{2}$ with the function

$$
F_{2}\left(x, Q^{2}\right)=a(x)\left[\ln \left(\frac{Q^{2}}{\Lambda^{2}}\right)\right]^{\kappa(x)} .
$$

Here $x$ is the Bjorken scale variable, $\Lambda$ is a scale parameter, chosen to be a typical value of the strong interaction scale $\Lambda=0.35 \mathrm{GeV}$. There are two free parameters: $a$ reflecting the hadronic structure at low momentum transfer $Q^{2}=e \cdot \Lambda^{2}=0.3 \mathrm{GeV}^{2}$, and $\kappa$ determining the positive and negative scaling violations of $F_{2}$. This allows an intuitive description of hadronic structures. It is the same ansatz that has been followed in the previous publication [1].

The analysis has been done using $F_{2}$ structure function measurements including most recent publications. They include proton structure function data and diffractive exchange data from the H1 and ZEUS experiments (HERA I) as well as new data of the photon hadronic structure measured at LEP (L3, ALEPH, OPAL and DELPHI experiments). The new data allow to decrease the errors in the determination of the hadronic structure parameter $a$ and the scaling violations parameter $\kappa$ substantially. In comparison with the previous publication the study has been extended to the charm contribution to proton structure function $F_{2}^{c \bar{c}}$ using data from the $\mathrm{H} 1$ and ZEUS experiments (HERA I, II) as well as EMC data.

The following analysis strategy has been pursued. To ensure that the measurements are dominated by deep inelastic lepton-hadron scattering with an underlying $F_{2}$ structure function the analysed data have been restricted to $Q^{2}>2 \mathrm{GeV}^{2}$. In addition the kinematic region has been limited by $Q^{2}<1000 \mathrm{GeV}^{2}$ and $y<0.6$.

For the fit procedure using equation (1.1) the combined statistical and systematic errors have been taken into account. For some measurements, e.g. for diffractive data, only reduced cross sections $\sigma_{r}^{D(3)}$ have been provided in the publications. In this case the structure function $F_{2}^{D(3)}$ has been calculated using $R=\frac{\sigma_{L}}{\sigma_{T}}=\frac{F_{L}}{F_{2}-F_{L}}=0.25$ taken from the $F_{L}$ proton measurements [2]].

For diffractive data the region with $x_{I P}<0.005$ has been considered which corresponds to the Pomeron-like region. In order to study the $Q^{2}$-dependence for a given $x$, an $x$-binning has been performed if not provided in the publication.

The fit has been done only for those $x$-bins for which more than two data points were available. For presenting the results of the fit the hadronic structure parameter $a$ and scaling violations parameter $\kappa$ have been plotted as a function of $x$ (see below). Only parameters with absolute errors of $\sigma_{a}<0.2$ and $\sigma_{\kappa}<1.0$ have been shown in the $a(x)$ and $\kappa(x)$ graphs.

\section{Comparison of the Hadronic Structures}

The fits for the proton structure function measurements are based on the data taken from the collider experiments ZEUS and H1 and from the fixed target experiment BCDMS [3, ఫ]. New $F_{2}$ data from HERA I run period have been taken from the combined H1 and ZEUS publication [3]. These data provide more precise measurements of hadronic structures for the intermediate and the low $x$ region. Data from $e^{+} p$ and $e^{-} p$ collisions have been fitted according to (1.1) separately for 

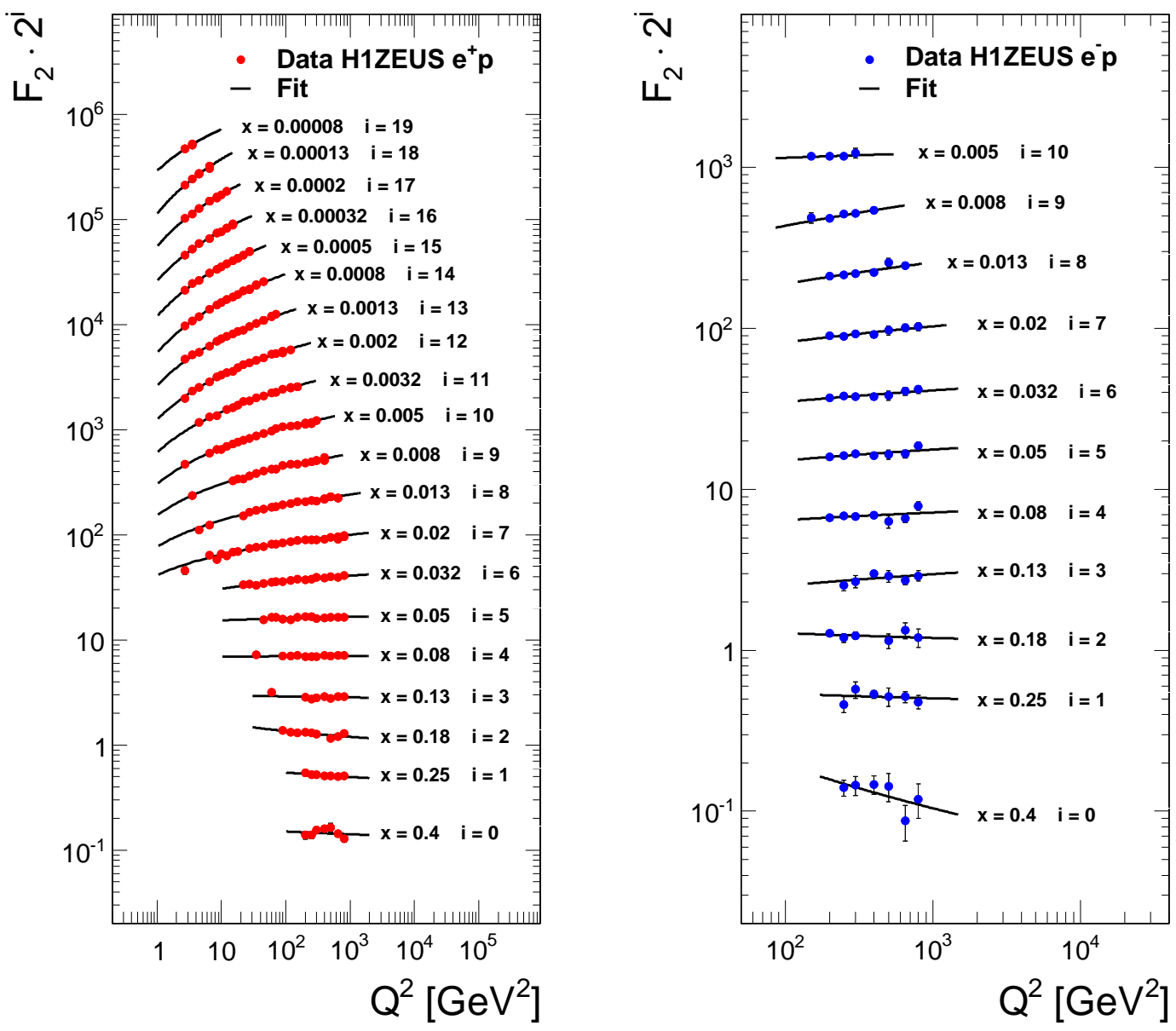

Figure 1: Two-parameter fit to the proton structure function $F_{2}$ for $e^{+} p$ (left figure) and $e^{-} p$ (right figure) data from the combined HERA I H1 and ZEUS measurements [3].

different $x$ bins (Figure 1). To cover the high $x$ region for the proton structure function the data of the BCDMS [ $₫$ ] fixed-target experiment have been analysed. The resulting fit parameters $a$ and $\kappa$ are shown in Figure (red full circles). One can see the valence quarks around $x \sim 0.3$. In the lower $x$ region the sea quarks dominate. A typical value for the hadronic structure at small $x$ is $a \sim 0.1$. Looking at the scaling violations, there is a continuous rise from the negative values starting in the high- $x$ region towards positive values for the low- $x$ (sea quark) region. The scaling violations are zero at $x \sim 0.1$.

The fits for the diffractive exchange are based on the ZEUS and H1 measurements of $F_{2}^{D(3)}$ at HERA I [5, 6, 7]. The parameters $a$ and $\kappa$ resulting from the fit to the colour singlet exchange data for the Pomeron-like region with $x_{I P}<0.005$ are shown in Figure 2 (magenta square symbols) as a function of $x=\beta$, the fraction of the colour singlet momentum carried by the interacting parton. In the upper figure the diffractive hadronic structure has been divided by a factor of 10 to enable its direct comparison with other hadronic structures. One can observe a high density at large parton momentum which can be explained by e.g. a two-gluon structure where one gluon carries a large 

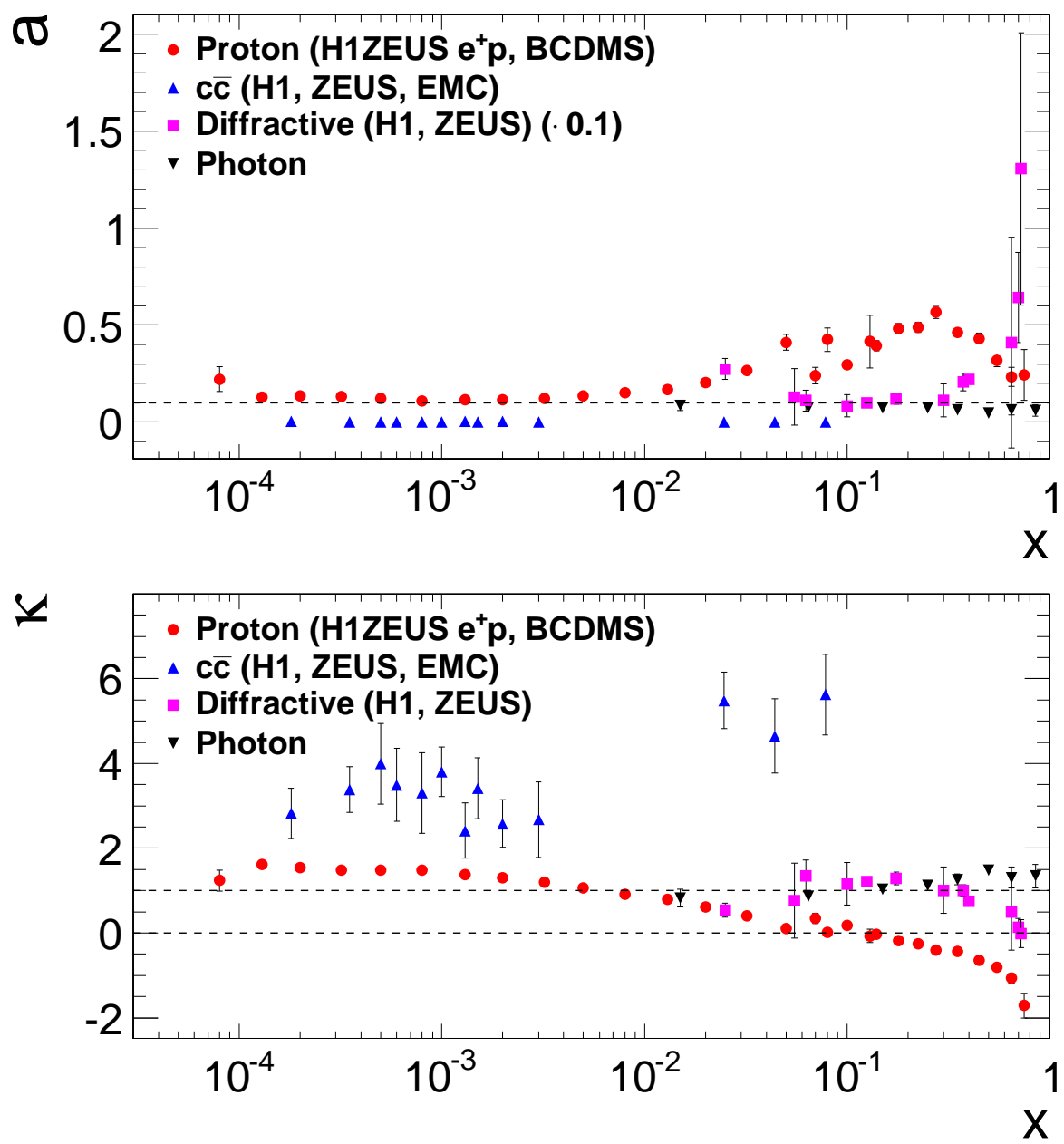

Figure 2: Hadronic structure parameter $a$ (upper figure) and scaling violations parameter $\kappa$ (lower figure) as results of the fit to proton, photon, diffractive exchange and proton charm contribution hadronic structures.

momentum fraction. The lower figure shows mostly large positive scaling violations which is, together with the large rate of diffractive events, suggestive of a gluon dominated exchange.

On the same footing it is possible to analyse the hadronic structure of the photon which results from the fluctuations of a photon into a colour neutral and flavour neutral hadronic state. For this purpose the new data from LEP as well as the data from JADE, TASSO and other experiments have been analysed [8]-[30]. Owing to the relatively small number of measurements in each individual experiment, their photon structure function data with systematic and statistic errors were used together in the fits. An $x$-binning has been done similar to [31] except for two first and two last bins in order to optimise the number of entries in each bin. In Figure 2 the fit parameters $a$ and $\kappa$ for the photon are shown (black triangular symbols). The hadronic structure of a photon does not show any evidence of valence quarks. The photon hadronic structure occurs from the fluctuations shortly before the collision. Like a proton at low values of $x$ the hadronic-like structure of a photon lies around $a \sim 0.1$. In contrast to a proton, a photon has only positive scaling violations with average 
value around $\kappa \sim 1$ as expected from QCD calculations [32].

In addition to afore mentioned objects the measurements of the charm contribution to the proton structure function $F_{2}^{c \bar{c}}$ have been analysed. One should pay attention that this component is already a part of the inclusive proton structure function $F_{2}$ analysed before. Having this component explicitly in hand it is interesting to compare it with other hadronic structures especially those ones originating from fluctuations. In this study the inclusive H1 HERA I, II measurements, Dmeson $\mathrm{H} 1$ and ZEUS HERA I data and EMC data have been analysed [33]-[38]. The fit results are shown in Figure (b (blue triangular symbols). The hadronic structure of the charm contribution to the proton structure is negligible when probing with the static resolution scale of $Q^{2}=0.3 \mathrm{GeV}^{2}$. There are strong positive scaling violations which is consistent with charm resulting from gluon fluctuations.

\section{Conclusion}

A wealth of new data from the $e p$ collisions at HERA and $e^{+} e^{-}$collisions at LEP allowed to perform an extensive comparison of hadronic structures extrapolated to $Q^{2}=0.3 \mathrm{GeV}^{2}$ (Figure 2). The proton hadronic structure shows valence quarks at $x \sim 0.3$ with sea quarks and negligible heavy quark contribution at low $x$. The photon has a similar level of the sea quarks as the proton. The hadronic structure of diffractive exchange reveals sea quarks at low $x$ and increases towards $x=1$ which is suggestive for two-gluon structure of a colour singlet object.

Scaling violations are strongly positive for the charm contribution to the proton structure, for the photon, the proton at low $x$ and diffractive exchange (except for $x=1$ ) and negative for the proton at high $x$.

\section{Acknowledgements}

We are very grateful for financial support of the Ministerium für Innovation,Wissenschaft, Forschung und Technologie des Landes Nordrhein-Westfalen, the Bundesministerium für Bildung und Forschung (BMBF), and the Helmholtz Alliance "Physics at the Terascale".

\section{References}

[1] M. Erdmann, Phys. Lett. B 488 (2000) 131 [arXiv:hep-ex/0007058].

[2] F. D. Aaron et al. [H1 Collaboration], Phys. Lett. B 665 (2008) 139 [arXiv:0805.2809 [hep-ex]].

[3] F. D. Aaron et al. [H1 Collaboration and ZEUS Collaboration], JHEP 1001 (2010) 109 [arXiv:0911.0884 [hep-ex]].

[4] A. C. Benvenuti et al. [BCDMS Collaboration], Phys. Lett. B 223 (1989) 485.

[5] S. Chekanov [ZEUS Collaboration], Nucl. Phys. B 800 (2008) 1 [arXiv:0802.3017 [hep-ex]].

[6] A. Aktas et al. [H1 Collaboration], Eur. Phys. J. C 48 (2006) 749 [arXiv:hep-ex/0606003].

[7] A. Aktas et al. [H1 Collaboration], Eur. Phys. J. C 48 (2006) 715 [arXiv:hep-ex/0606004].

[8] M. Acciarri et al. [L3 Collaboration], Phys. Lett. B 436 (1998) 403. 
[9] M. Acciarri et al. [L3 Collaboration], Phys. Lett. B 447 (1999) 147.

[10] M. Acciarri et al. [L3 Collaboration], Phys. Lett. B 483 (2000) 373 [arXiv:hep-ex/0004005].

[11] P. Achard et al. [L3 Collaboration], Phys. Lett. B 622 (2005) 249 [arXiv:hep-ex/0507042].

[12] R. Akers et al. [OPAL Collaboration], Z. Phys. C 61 (1994) 199.

[13] K. Ackerstaff et al. [OPAL Collaboration], Z. Phys. C 74 (1997) 33.

[14] K. Ackerstaff et al. [OPAL Collaboration], Phys. Lett. B 411 (1997) 387 [arXiv:hep-ex/9708019].

[15] K. Ackerstaff et al. [OPAL Collaboration], Phys. Lett. B 412 (1997) 225 [arXiv:hep-ex/9708028].

[16] G. Abbiendi et al. [OPAL Collaboration], Eur. Phys. J. C 18 (2000) 15 [arXiv:hep-ex/0007018].

[17] R. Barate et al. [ALEPH Collaboration], Phys. Lett. B 458 (1999) 152.

[18] A. Heister et al. [ALEPH Collaboration], Eur. Phys. J. C 30 (2003) 145.

[19] P. Abreu et al. [DELPHI Collaboration], Z. Phys. C 69 (1996) 223.

[20] T. Sasaki et al. [AMY Collaboration], Phys. Lett. B 252 (1990) 491.

[21] S. K. Sahu et al. [AMY Collaboration], Phys. Lett. B 346 (1995) 208.

[22] T. Kojima et al. [AMY Collaboration], Phys. Lett. B 400 (1997) 395.

[23] K. Muramatsu et al. [TOPAZ Collaboration], Phys. Lett. B 332 (1994) 477.

[24] M. Althoff et al. [TASSO Collaboration], Z. Phys. C 31 (1986) 527.

[25] W. Bartel et al. [JADE Collaboration], Z. Phys. C 24 (1984) 231.

[26] W. Bartel et al. [JADE Collaboration], Phys. Lett. B 121 (1983) 203.

[27] C. Berger et al. [PLUTO Collaboration], Phys. Lett. B 142 (1984) 111.

[28] C. Berger et al. [PLUTO Collaboration], Nucl. Phys. B 281 (1987) 365.

[29] H. Aihara et al. [TPC/Two Gamma Collaboration], Z. Phys. C 34 (1987) 1.

[30] H. Aihara et al. [TPC/Two Gamma Collaboration], Phys. Rev. Lett. 58 (1987) 97.

[31] R. Nisius, arXiv:0907.2782 [hep-ex].

[32] E. Witten, Nucl. Phys. B 120 (1977) 189.

[33] C. Adloff et al. [H1 Collaboration], Phys. Lett. B 528 (2002) 199 [arXiv:hep-ex/0108039].

[34] F. D. Aaron et al. [H1 Collaboration], Eur. Phys. J. C 65 (2010) 89 [arXiv:0907.2643 [hep-ex]].

[35] S. Chekanov et al. [ZEUS Collaboration], Phys. Rev. D 69 (2004) 012004 [arXiv:hep-ex/0308068].

[36] A. Aktas et al. [H1 Collaboration], Eur. Phys. J. C 40 (2005) 349 [arXiv:hep-ex/0411046].

[37] A. Aktas et al. [H1 Collaboration], Eur. Phys. J. C 45 (2006) 23 [arXiv:hep-ex/0507081].

[38] J. J. Aubert et al. [European Muon Collaboration], Nucl. Phys. B 213 (1983) 31. 